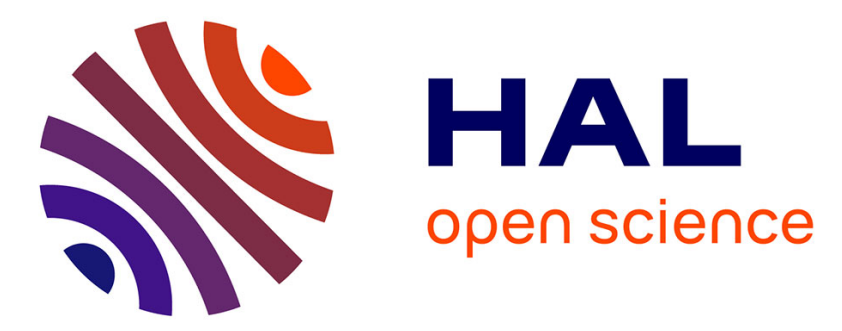

\title{
Urban Traffic Eco-driving: A Macroscopic Steady-State Analysis
}

Giovanni de Nunzio, Carlos Canudas de Wit, Philippe Moulin

\section{To cite this version:}

Giovanni de Nunzio, Carlos Canudas de Wit, Philippe Moulin. Urban Traffic Eco-driving: A Macroscopic Steady-State Analysis. ECC 2014 - 13th European Control Conference, Jun 2014, Strasbourg, France. 10.1109/ECC.2014.6862403 . hal-01022713

\section{HAL Id: hal-01022713 https://hal.inria.fr/hal-01022713}

Submitted on 10 Jul 2014

HAL is a multi-disciplinary open access archive for the deposit and dissemination of scientific research documents, whether they are published or not. The documents may come from teaching and research institutions in France or abroad, or from public or private research centers.
L'archive ouverte pluridisciplinaire HAL, est destinée au dépôt et à la diffusion de documents scientifiques de niveau recherche, publiés ou non, émanant des établissements d'enseignement et de recherche français ou étrangers, des laboratoires publics ou privés. 


\title{
Urban Traffic Eco-driving: A Macroscopic Steady-State Analysis
}

\author{
Giovanni De Nunzio, Carlos Canudas de Wit, Philippe Moulin
}

\begin{abstract}
The problem of traveling at maximum energy efficiency (Eco-Driving) is addressed for urban traffic networks at macroscopic level. The scope of this paper is the analysis of the steady-state behavior of the system, given certain boundary flows conditions fixed by traffic lights timings, and in presence of a traffic control policy based on variable speed limits. The formal study is carried out on a two-cells variable length model adapted to the urban setup from previous works on highway traffic [1][2]. Informative traffic metrics, aimed at assessing traffic and vehicles performance in terms of traveling time, infrastructure utilization and energy consumption, are then defined and adapted to the new macroscopic traffic model.

If congestion in a road section does not spill back or vanish, the system is stable and many different equilibrium points can be reached via variable speed limits. Efficient operation points and traffic conditions are identified as a trade-off between optimization of global traffic energy consumption, traveling time and infrastructure utilization.
\end{abstract}

Index Terms-Traffic energy, velocity planning, variable speed limits, traffic lights.

\section{INTRODUCTION}

The problem of traffic congestion on urban roads is of great public interest due to its influence on security and pollution levels. Therefore it is of primary importance to have tools to easily and effectively model and control traffic evolution, as well as find optimal traffic configurations in terms of energy consumption and other performance metrics.

Macroscopic traffic models, as opposed to microscopic models, are very appealing for their simplicity and accuracy in describing traffic evolution as a fluid in a pipe, by considering vehicles flows and densities. Many efforts have been channeled by scientific community into analyzing highway traffic from a macroscopic point of view, and the focus on modeling urban traffic networks is relatively recent.

The existence of a macroscopic fundamental diagram for urban roads with no turns and regulated by traffic signals has been proven in [3], where the authors also provided some experimental results. Therefore different types of macroscopic models adapted to the urban case have been proposed. Models describing only queues length have been proposed in [4][5] for the sake of simplicity and speed of the predictive control strategy. However very often these control strategies require long sampling times for the online implementation, and the queue length as only state variable does not allow

G. De Nunzio, Ph.D. Candidate at GIPSA-Lab and IFPen, France, giovanni.de-nunzio@ifpen.fr

C. Canudas de Wit, Director of Research at CNRS, GIPSA-Lab, NeCS team, Grenoble, France, carlos.canudas-de-wit@gipsa-lab.fr

P. Moulin, Department of Control Signal and Systems at IFPen, RueilMalmaison, France, philippe.moulin@ifpen.fr

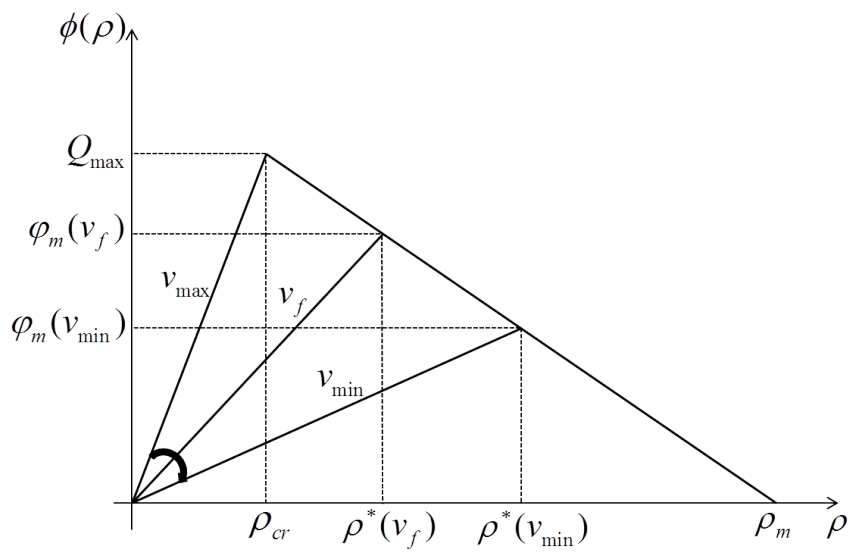

Fig. 1: Fundamental diagram with variable speed limits.

satisfactory assessment of the energy consumption in the whole considered road stretch. The Cell Transmission Model (CTM), originally proposed in [6], has also been adapted to the urban environment. In [7][8], the authors extend the original CTM by considering the effects of turnings and channelization at the downstream end of a road section, and a different computation of boundary flows is proposed according to the position of the considered cell in the section. In [9], the well-known CTM problem of poor accuracy in the case of too long cells is addressed by improving the computation of boundary flows with the information about queue length, showing already the intuition of the necessity of congestion length information for accurate description of traffic evolution in a section.

Given the macroscopic model to describe traffic evolution, the problem of computing traffic energy consumption is critical and challenging. Many works have addressed this topic on a highway setting proposing different strategies. In [10], starting from a second order macroscopic model with densities and velocities as state variables, the emissions cost function is defined as a nonlinear polynomial function of the average velocities in the different sectors. As opposed to these average-speed based emissions models, dynamic energy consumption models make use of motion variables coming from individual vehicles. Within the objective of combining simplicity of the macroscopic traffic models with the accuracy of microscopic energy consumption models, one of the main problems is how to reconstruct acceleration profiles from macroscopic variables. In [11][12], the authors approximate accelerations from the velocities returned by the second order model (METANET), by considering the speed 
difference between successive time steps, and plug them into the fuel consumption model VT-micro.

The contribution of this work is twofold. Firstly, at modeling level, the Variable Length Model (VLM), proposed in [1][2], is adapted and extended to the considered urban traffic environment, by modeling boundary flows affected by both traffic lights and a variable-speed-limits control structure. This model offers important advantages with respect to other first order or second order macroscopic models, since it allows to accurately describe the traffic evolution in a road section with only two cells and a variable representing the moving front of congestion. The cyclic nature of the traffic lights and system behavior allows finally to adopt an average representation of the model by means of the averaging method for periodic systems, in order to have a better suited framework for analysis and control. A macroscopic energy consumption model and known traffic performance metrics have been adapted to the VLM in order to assess traffic behavior. Secondly, at analysis level, a steady-state evaluation of the traffic conditions is carried out in order to identify the best operation points in terms of collective energy consumption and other defined traffic metrics, and to understand what control action can be designed. The objective is to bring the system to a certain equilibrium by means of variable speed limits such that energy consumption is minimized in trade-off with important traffic metrics such as travel time and travel distance. This type of control action, which is supposed to be followed by all the vehicles in the network, is made feasible by the new available technologies in intelligent transportation systems ( $\mathrm{I} 2 \mathrm{~V}$ and $\mathrm{V} 2 \mathrm{~V}$ communication, advanced driver assistance systems, etc.).

In section II the Variable Length Model is presented and appropriately modified for the analyzed framework, in section III traffic metrics and energy consumption model are defined and adapted to the VLM, in section IV a steadystate analysis of the possible operation points is performed, conclusions and remarks in section $\mathrm{V}$.

\section{VARIABLE LENGTH MODEL FOR URBAN NetWORKS}

The first instance of a macroscopic traffic model, intended to solve the kinematic wave equation, was the LWR model introduced in [13][14], which is a continuous first order model of the form:

$$
\frac{\partial}{\partial t} \rho+\frac{\partial}{\partial x} \phi(\rho)=0
$$

where $\rho$ indicates the density of vehicles, $\phi(\rho)=\rho v$ is the flux of vehicles and $v$ is their average velocity.

The Cell Transmission Model (CTM), introduced by Daganzo in [6], is a well known and widely used discretized version of the LWR, and in particular it is its first order Godunov approximation. The main idea of the CTM is to divide the road section under analysis into a certain number of homogeneous cells. The key assumption, motivated by experimental data, is that the vehicles travel at an equilibrium speed $v=v(\rho)$. Since the flow is defined as $\phi(\rho)=\rho v(\rho)$, one can represent an equilibrium flow function $\varphi=\phi(\rho)$ called macroscopic fundamental diagram.

Despite its versatility in modeling highway traffic, CTM presents some critical drawbacks in realistically depicting density evolution and distribution when the discretization is not fine enough (i.e. small number of too long cells) or when congestion arises.

\section{A. Ground Basis of Variable Length Model}

The Variable Length Model (VLM), originally proposed in [1] and further modified in [2], provides a better representation of the congestion, which in urban networks is very likely to arise due to the presence of traffic lights. The number of cells per section is reduced to two and each road section is modeled with only three state variables: density in the upstream free cell $\rho_{f}$, density in the downstream congested cell $\rho_{c}$, position of the congestion front $l$. Consider a road section of length $L$, then the free cell's length will be $(L-l)$ and the congested cell's length $l$. Density of vehicles in the two lumped cells is averaged, which means notion of singlevehicle behavior is lost. The dynamic equations of the VLM are derived from the vehicles conservation principle:

$$
\frac{\mathrm{d}}{\mathrm{d} t} N=\varphi_{\text {in }}-\varphi_{\text {out }}, \quad N=\int_{0}^{L} \rho(x, t) \mathrm{d} x
$$

where $N$ is the number of vehicles, $\varphi_{\text {in }}$ and $\varphi_{\text {out }}$ are the inflow and outflow at the boundaries of the section of length $L$. In the VLM setting, the number of vehicles is defined as:

$$
N=\rho_{f}(L-l)+\rho_{c} l
$$

The domain of existence of the densities in the two cells is:

$$
\begin{array}{r}
\rho_{f} \in\left[0, \rho^{*}\left(v_{f}\right)\right] \\
\rho_{c} \in\left(\rho^{*}\left(v_{f}\right), \rho_{m}\right]
\end{array}
$$

where $\rho^{*}\left(v_{f}\right)$ is the critical density relative to the current maximum allowed speed in the free cell:

$$
\rho^{*}\left(v_{f}\right)=\frac{w \rho_{m}}{v_{f}+w}
$$

$v_{f}$ may be thought of as the current maximum speed limit in the free cell and would be utilized as the control input in a variable speed limit traffic controller, and $\rho_{m}$ is the jam density of the road section (see Fig.1).

The dynamic equations of the model are as follows ${ }^{1}$ :

$$
\Sigma_{1}:\left\{\begin{array}{l}
\dot{\rho}_{f}=\left[\varphi_{\mathrm{in}}-\phi\left(\rho_{f}\right)\right] \frac{1}{L-l} \\
\dot{\rho}_{c}=\left[\phi\left(\rho_{c}\right)-\varphi_{\text {out }}\right] \frac{1}{l} \\
\dot{l}=\frac{\phi\left(\rho_{f}\right)-\phi\left(\rho_{c}\right)}{\rho_{c}-\rho_{f}}
\end{array}\right.
$$

\footnotetext{
${ }^{1}$ The system structure described by (6) is an improvement of the model presented in [1]. This new formalization of the model, described in [2], has invariance properties by construction, that is the two state variables $\rho_{f}$ and $\rho_{c}$ remain in their domain of existence (4), assuming that initial conditions are taken in the domain of existence.
} 
The boundary flows are defined as:

$$
\begin{aligned}
& \varphi_{\text {in }}=\min \left\{D_{\text {in }}, S_{f}\right\} \\
& \varphi_{\text {out }}=\min \left\{D_{c}, S_{\text {out }}\right\}
\end{aligned}
$$

where $D_{c}$ and $S_{f}$ are:

$$
\begin{aligned}
& D_{c}=\min \left\{\rho_{c} v_{f}, \varphi_{m}\left(v_{f}\right)\right\} \\
& S_{f}=\min \left\{w\left(\rho_{m}-\rho_{f}\right), \varphi_{m}\left(v_{f}\right)\right\}
\end{aligned}
$$

Assuming that the two cells have the same fundamental diagram, the saturation function for demand and supply depends on the maximum allowed speed limit:

$$
\varphi_{m}\left(v_{f}\right)=\rho^{*}\left(v_{f}\right) v_{f}=\frac{w \rho_{m}}{v_{f}+w} v_{f}
$$

$D_{\text {in }} \leq Q_{\max }$ is the input demand, $S_{\text {out }} \leq Q_{\max }$ is the output supply, and $Q_{\max }$ is the maximum capacity of the road section which, once fixed maximum allowed velocity $\left(v_{\max }\right)$, back-propagation speed of the congestion $(w)$ and jam density $\left(\rho_{m}\right)$, is uniquely defined after the nominal critical density as:

$$
\rho_{\mathrm{cr}}=\frac{w \rho_{m}}{v_{\max }+w}, \quad Q_{\max }=\rho_{c r} v_{\max }
$$

The interface flows $\phi\left(\rho_{f}\right)$ and $\phi\left(\rho_{c}\right)$, which correspond to the demand of the free cell and the supply of the congested cell respectively, due to the invariance properties of the system may be simply defined as:

$$
\begin{aligned}
& \phi\left(\rho_{f}\right)=D_{f}=\rho_{f} v_{f} \\
& \phi\left(\rho_{c}\right)=S_{c}=w\left(\rho_{m}-\rho_{c}\right)
\end{aligned}
$$

\section{B. Adaptation of VLM to the urban environment}

Let us assume that the urban network under analysis is divided into $n$ sections separated by traffic lights. The switching variable $\alpha(t)$ models the behavior of traffic lights (Fig.2), and it obviously regulates the outflow of the section acting like a valve enabling and disabling the output stream of vehicles. The modeling variable takes on binary values according to the current phase of the traffic light, it is cycletime $\left(T_{\text {cycle }}\right)$ periodic and it is simply defined as:

$$
\alpha(t)=\alpha\left(t+T_{\text {cycle }}\right)= \begin{cases}1, & \text { if } t \leq \tau \leq t+T_{\mathrm{gr}} \\ 0, & \text { if } t+T_{\mathrm{gr}}<\tau<t+T_{\text {cycle }}\end{cases}
$$

where $T_{\mathrm{gr}}$ and $T_{\text {cycle }}$ represent the green phase time and the cycle time of the traffic light, respectively.

Then the boundary flows of section $i \in[1, n]$, applying the known demand-supply formalism for merging traffic, vary depending on the position of the considered section in the network and on the current speed limit.

Inflow for the section $i$ is now defined as:

$$
\varphi_{\text {in }, \alpha}\left(t, v_{f}\right)=\alpha_{i-1}(t) \cdot \min \left\{D_{\text {in }}, S_{f}\right\}
$$

and the input demand, assuming that a queue is present before every traffic light, is:

$$
D_{\text {in }}=\left\{\begin{array}{l}
\varphi_{m}\left(v_{f, i-1}\right), \quad \text { if } i=1 \\
\min \left\{D_{c, i-1}, \varphi_{m}\left(v_{f, i-1}\right)\right\}, \quad \text { otherwise }
\end{array}\right.
$$

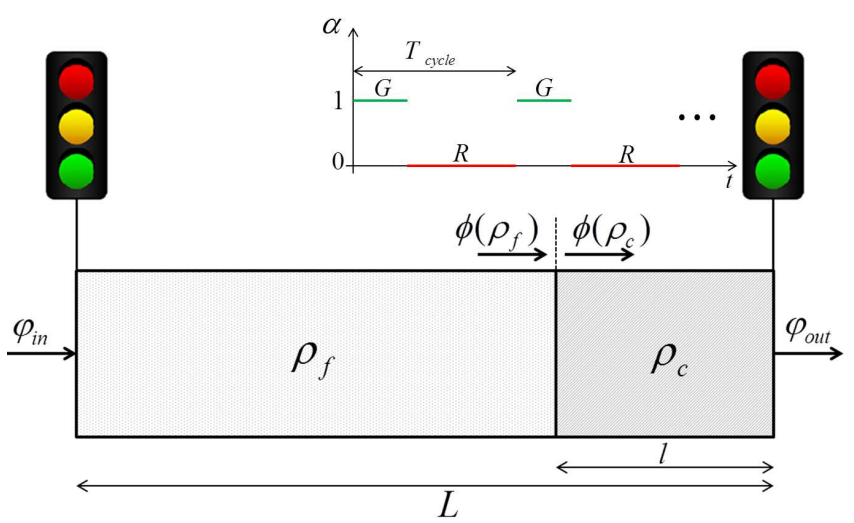

Fig. 2: Urban section scheme with model variables.

Outflow is defined as:

$$
\varphi_{\text {out }, \alpha}\left(t, v_{f}\right)=\alpha(t) \cdot \min \left\{D_{c}, S_{\text {out }}\right\}
$$

and the output supply is:

$$
S_{\text {out }}=\left\{\begin{array}{l}
\varphi_{m}\left(v_{f, i+1}\right), \quad \text { if } i=n \\
\min \left\{S_{f, i+1}, \varphi_{m}\left(v_{f, i+1}\right)\right\}, \quad \text { otherwise }
\end{array}\right.
$$

For each section of the urban network, system $\Sigma_{1}$ in (6) now can be rewritten as:

$$
\Sigma_{2}:\left\{\begin{array}{l}
\dot{\rho}_{f}=\left[\varphi_{\mathrm{in}, \alpha}\left(t, v_{f}\right)-\rho_{f} v_{f}\right] \frac{1}{L-l} \\
\dot{\rho}_{c}=\left[w\left(\rho_{m}-\rho_{c}\right)-\varphi_{\mathrm{out}, \alpha}\left(t, v_{f}\right)\right] \frac{1}{l} \\
i=\frac{\rho_{f} v_{f}-w\left(\rho_{m}-\rho_{c}\right)}{\rho_{c}-\rho_{f}}
\end{array}\right.
$$

\section{Averaged VLM}

An interesting simplification of this model for control purposes, to avoid the binary behavior of the switching variable modeling the traffic light, is obtained and formally justified with the averaging theory [15]. Let us consider a system of the form

$$
\dot{x}=\epsilon f(t, x, \epsilon)
$$

where $f$ is differentiable with respect to $(x, \epsilon)$ up to the second order and it is $\Delta T$-periodic in $t$, then we associate with (18) an autonomous average system

$$
\dot{x}=\epsilon f_{a v}(x)
$$

where

$$
f_{a v}(x)=\frac{1}{\Delta T} \int_{t}^{t+\Delta T} f(\tau, x, 0) \mathrm{d} \tau
$$

System $\Sigma_{2}$ satisfies the hypotheses of the averaging theory since $f(x) \in \mathcal{C}^{2}$, and only the boundary flows $\varphi_{\mathrm{in}, \alpha}\left(t, v_{f}\right)$ and $\varphi_{\text {out }, \alpha}\left(t, v_{f}\right)$ depend on the purely time-dependent variable $\alpha(t)$. Therefore, the traffic lights behavior can be averaged over the period (i.e. cycle time) as follows:

$$
\bar{\alpha}=\frac{1}{T_{\text {cycle }}} \int_{t}^{t+T_{\text {cycle }}} \alpha(\tau) \mathrm{d} \tau=\frac{1}{T_{\text {cycle }}} \int_{t}^{t+T_{\mathrm{gr}}} \mathrm{d} \tau=\frac{T_{\mathrm{gr}}}{T_{\text {cycle }}}
$$


Hence, averaging method applied to system $\Sigma_{2}$, yields boundary flows defined as:

$$
\begin{aligned}
& \bar{\varphi}_{\text {in }}\left(v_{f}\right)=\bar{\alpha}_{i-1} \cdot \min \left\{D_{\text {in }}, S_{f}\right\} \\
& \bar{\varphi}_{\text {out }}\left(v_{f}\right)=\bar{\alpha} \cdot \min \left\{D_{c}, S_{\text {out }}\right\}
\end{aligned}
$$

and the new average system formulation, which will be used as the reference model in the following analysis, is:

$$
\Sigma_{3}:\left\{\begin{array}{l}
\dot{\bar{\rho}}_{f}=\left[\bar{\varphi}_{\text {in }}-\rho_{f} v_{f}\right] \frac{1}{L-l} \\
\dot{\bar{\rho}}_{c}=\left[w\left(\rho_{m}-\rho_{c}\right)-\bar{\varphi}_{\text {out }}\right] \frac{1}{l} \\
\dot{\bar{l}}=\frac{\rho_{f} v_{f}-w\left(\rho_{m}-\rho_{c}\right)}{\rho_{c}-\rho_{f}}
\end{array}\right.
$$

This simplification is also consistent with the store-andforward modeling approach [16]. The oscillatory behavior of the system, induced by the traffic lights, is lost and the vehicles entering or leaving the section may be seen as a continuous flow passing through a bottleneck, as long as demand and supply functions of upstream and downstream cells are capacious enough. As natural consequences of this approximation, no oscillations of the congestion length, due to the green/red alternation, are represented by the model, and the notions of cycle time and offset for the traffic lights lose significance.

\section{Traffic Performance Metrics}

Traffic conditions need to be evaluated and assessed with respect to performance metrics properly defined and adapted to the employed model $\Sigma_{3}$. It has to be recalled that the interest of this work is in the steady-state analysis of the system, therefore the performance metrics, for consistency, will be evaluated over a period of the traffic light $\left(T_{\text {cycle }}\right)$ at steady-state.

\section{A. Instantaneous Travel Time}

Instantaneous Travel Time (ITT) may be defined as the travel time that would result if traffic conditions remained unchanged over the time span under analysis, and, for a single vehicle, it is:

$$
\operatorname{ITT}(\rho)=\frac{L-l}{v_{f}}+\frac{l}{v_{c}}
$$

where velocity in a cell is a function of the density in that cell and it is given by the general relationship [6]:

$$
v(\rho)= \begin{cases}v_{f} & \text { if } \rho \leq \rho^{*}\left(v_{f}\right) \\ -w\left(1-\frac{\rho_{m}}{\rho}\right) & \text { otherwise }\end{cases}
$$

\section{B. Total Travel Time}

Total Travel Time (TTT) is one of the most used and informative global traffic metrics to assess nature of traffic and vehicles behavior, besides being a socially critical measure to be minimized. If ITT gives a step-by-step quantification of the time a single vehicle would spend in the section, TTT is global and it is only influenced by the evolution of the number of vehicles inside the section over a certain time horizon T. Total Travel Time for the VLM, over the traffic light cycle time at steady state, is:

$$
\operatorname{TTT}(\rho)=\int_{0}^{T} \int_{0}^{L} \rho(\tau) \mathrm{d} x \mathrm{~d} \tau=\int_{t}^{t+T_{\text {cycle }}}\left[\rho_{f} L+\left(\rho_{c}-\rho_{f}\right) l\right] \mathrm{d} \tau
$$

Minimization of total time spent in a traffic network is equivalent to maximization of flow. In other words, the earlier the vehicles are able to exit the network (by appropriate use of the available control measures) the smaller TTT will be [16]. It is also important to notice that if reducing $v_{f}$ does not induce a reduction of the inflow, the only effect would be a redistribution of the vehicles inside the road section, the average number of vehicles would not change and the TTT would not be affected.

\section{Total Travel Distance}

Total Travel Distance (TTD) is a measure of how efficiently the infrastructure is used in terms of occupancy and traveling velocity. The infrastructure holder would like to maximize this metric in order to have as many vehicles as possible traveling at the maximum allowed velocity, that is having a high utilization of the infrastructure with no congestion. It is adapted to VLM and evaluated over the traffic light cycle time as:

$$
\begin{aligned}
\operatorname{TTD}(\rho) & =\int_{t}^{t+T_{\text {cycle }}} \int_{0}^{L} \phi(\rho, \tau) \mathrm{d} x \mathrm{~d} \tau \\
& =\int_{t}^{t+T_{\text {cycle }}} \int_{0}^{L-l} \phi\left(\rho_{f}, \tau\right) \mathrm{d} x \mathrm{~d} \tau+\int_{t}^{t+T_{\text {cycle }}} \int_{L-l}^{L} \phi\left(\rho_{c}, \tau\right) \mathrm{d} x \mathrm{~d} \tau \\
& =\int_{t}^{t+T_{\text {cycle }}} \int_{0}^{L-l} v_{f} \rho_{f} \mathrm{~d} x \mathrm{~d} \tau+\int_{t}^{t+T_{\text {cycle }}} \int_{L-l}^{L} w\left(\rho_{m}-\rho_{c}\right) \mathrm{d} x \mathrm{~d} \tau \\
& =\int_{t}^{t+T_{\text {cycle }}}\left\{v_{f} \rho_{f} L+\left[w\left(\rho_{m}-\rho_{c}\right)-v_{f} \rho_{f}\right] l\right\} \mathrm{d} \tau
\end{aligned}
$$

\section{Energy}

Another important metric, usually not considered at macroscopic level, is the energy consumption of the vehicles. The energy consumption functional here is obtained as an adaptation to the macroscopic case of the one in [17], assuming that the vehicles in the traffic network are all equipped with an electric motor (analogous metrics like the VT-macro [11] could be used in the case of vehicles with combustion engines). It is recalled that for a single vehicle the model is written as:

$$
\left\{\begin{array}{l}
\dot{x}=v \\
\dot{v}=h_{1} u-h_{2} v^{2}-h_{3} v-h_{0}
\end{array}\right.
$$


where parameters $h$ are estimated for the electric motor under analysis [18] and the single-vehicle control input (i.e. motor torque), for constant-speed trips (i.e. average velocity $\bar{v}$ is constant within the cells), is:

$$
\bar{u}=\frac{1}{h_{1}}\left(h_{2} \bar{v}^{2}+h_{3} \bar{v}+h_{0}\right)
$$

Then power demand for an electric motor is given by:

$$
P=b_{1} u v+b_{2} u^{2}=f(v, \dot{v})
$$

where $b_{1}$ and $b_{2}$ are motor parameters defined in [17], and for the particular case of constant-speed trips:

$$
\bar{P}=b_{1} \bar{u} \bar{v}+b_{2} \bar{u}^{2}
$$

Hence the energy functional may be generally written as:

$$
E=\int_{0}^{T} P \mathrm{~d} \tau
$$

At a macroscopic level, the energy consumption is affected by the number of vehicles traveling in the road section under analysis, along with velocity and acceleration of the vehicles embedded in traffic flow. Therefore, by tailoring the energy cost functional to the VLM, energy consumption may be approximated as the sum of different contributions: energy consumption in the free cell, energy consumption in the congested cell, energy consumption at the interface points between two adjacent cells. Velocity of the vehicles is given by (25) and acceleration at the jump points (interfaces between different cells) can be simply modeled as a constant:

$$
a=\min \left\{a_{\max }, \frac{\Delta v}{\Delta k}\right\}
$$

where $\Delta k$ is the discretization step, and $\Delta v$ can be either $\left(v_{c}-v_{f}\right)$ if the acceleration is the one at the interface between the free and the congested cell of the considered section, or $\left(v_{f, i+1}-v_{c}\right)$ if the acceleration is the one applied to leave the section downstream. Parameter $a_{\max }$ indicates the maximum acceleration a driver is going to apply and it can be fixed according to safety and/or comfort policies.

Finally, the energy cost functional, over a time horizon $T_{\text {cycle, }}$, for one section for the VLM can be formulated as follows:

$$
E=E_{f}+E_{c}+E_{f \rightarrow c}+E_{c \rightarrow f}
$$

where energy consumption in the free cell for a constant velocity $\left(v_{f}\right)$ trip is:

$$
E_{f}=\int_{t}^{t+T_{\text {cycle }}} \bar{P}_{f}(\tau) \cdot \rho_{f}(\tau) \cdot(L-l(\tau)) \mathrm{d} \tau
$$

energy consumption in the congested cell for a constant velocity $\left(v_{c}\right)$ trip is:

$$
E_{c}=\int_{t}^{t+T_{\text {cycle }}} \bar{P}_{c}(\tau) \cdot \rho_{c}(\tau) \cdot l(\tau) \mathrm{d} \tau
$$

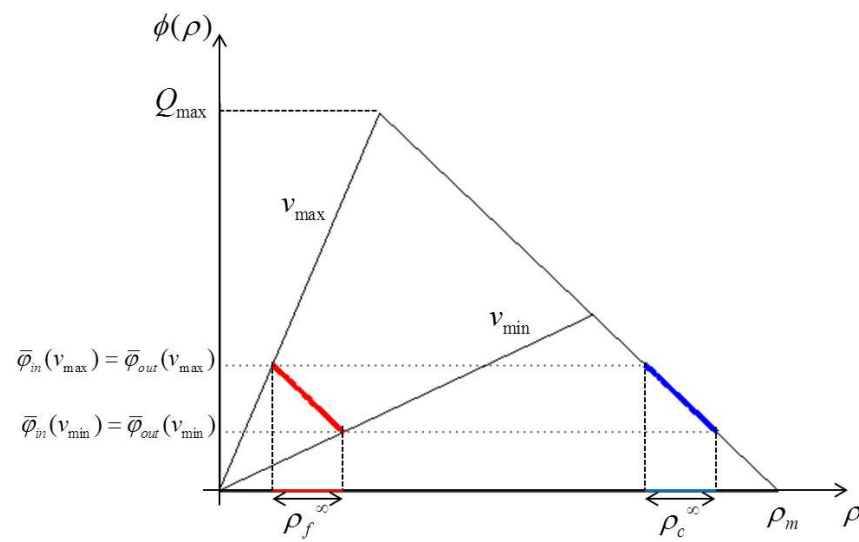

Fig. 3: Range of possible operation points reachable at steady-state via variable speed limits. $v_{\max }$ and $v_{\min }$ are given.

energy consumption due to the velocity change between the free and the congested cell is:

$$
E_{f \rightarrow c}=\int_{t}^{t+T_{\text {cycle }}} P(\tau) \cdot \rho_{f}(\tau) \cdot v_{f}(\tau) \Delta k \mathrm{~d} \tau
$$

and analogously energy consumption due to the velocity change between the congested and the free cell downstream of the traffic light is:

$$
E_{c \rightarrow f}=\int_{t}^{t+T_{\text {cycle }}} P(\tau) \cdot \rho_{c}(\tau) \cdot v_{c}(\tau) \Delta k \mathrm{~d} \tau
$$

Note that in the power demand expression (30), used to approximate energy consumption at the interfaces, $\dot{v}$ is assumed to be equal to $a$ in (33) and $v$ can be written as:

$$
v(t)=v_{0}+a \cdot t
$$

where $v_{0}$ will be either $v_{f}$ or $v_{c}$ depending on the considered transition.

\section{OPTIMAL STEADY-STATE VELOCITY}

\section{A. Problem formulation}

The goal of this section is to analyze the steady-state behavior of the system and select the velocity in the free cell, via variable speed limits, which minimizes an objective function depending on energy consumption, ITT, TTT, TTD. As working hypotheses, the scenario under analysis takes into account one road section with two traffic lights regulating the boundary flows. Upstream and downstream the considered section there supposed to be enough demand and supply and the traffic lights' split ratios are set to be equal. This makes the control problem more interesting due to the presence of many possible steady-state operation points (Fig.3).

Problem 1: Given system $\Sigma_{3}$ and a constant $\bar{\alpha}$ for every traffic light, find the optimal speed limit

$$
v_{f}^{*}=\underset{v_{f}}{\operatorname{argmin}}\left\{E+\sigma_{1} \mathrm{ITT}+\sigma_{2} \mathrm{TTT}-\sigma_{3} \mathrm{TTD}\right\}
$$


under

$$
\begin{aligned}
& v_{f} \in \mathcal{U}_{v}=\left\{v_{f}: v_{\min } \leq v_{f} \leq v_{\max }\right\} \\
& l \in \mathcal{U}_{l}=\{l: 0 \leq l \leq L\}
\end{aligned}
$$

As the model is defined, it results evident that if there is a difference in the boundary flows the system converges naturally to either fully free or fully congested state. If the system is in free state, Problem 1 is still well posed and would be simpler since energy consumption would be given only by the $E_{f}$ contribution. On the contrary if the system is in congested state, no control action can be applied in the free cell at steady-state to prevent congestion from increasing and eventually saturating the section.

Varying the speed limit in the free cell, which is the only controllable part of the section, will cause the system to reach a different steady-state value without modifying the number of vehicles, hence complying with the hypothesis of equal boundary flows. This corresponds to just a redistribution of vehicles inside the section, changing the number of vehicles in the free cell and congested cell while moving $l$.

Result 1: Holding the hypothesis of constant and equal boundary flows smaller than maximum flow, and given a set of initial condition $\left(\rho_{f}^{0}, \rho_{c}^{0}, l^{0}\right)$, such a system converges to the following equilibrium states (see Fig.3):

$$
\begin{aligned}
& \rho_{f}^{\infty}=\frac{\bar{\varphi}_{\text {in }}}{v_{f}} \\
& \rho_{c}^{\infty}=\rho_{m}-\frac{\bar{\varphi}_{\text {out }}}{w} \\
& l^{\infty}=\frac{N_{0}-\rho_{f}^{\infty} L}{\rho_{c}^{\infty}-\rho_{f}^{\infty}}=\frac{w v_{f} N_{0}-\bar{\varphi}_{\text {in }} L w}{w v_{f} \rho_{m}-\bar{\varphi}_{\text {out }} v_{f}-\bar{\varphi}_{\text {in }} w}
\end{aligned}
$$

where $N_{0}$, the initial number of vehicles, is calculated as:

$$
N_{0}=\rho_{f}^{0}\left(L-l^{0}\right)+\rho_{c}^{0} l^{0}
$$

Since the global metric TTT is not impacted by regulation via variable speed limits under the problem's hypotheses, Problem 1 results to be equivalent to the simpler problem:

Problem 2: Given system $\Sigma_{3}$ and a constant $\bar{\alpha}$ for every traffic light, find the optimal speed limit

$$
v_{f}^{*}=\underset{v_{f}}{\operatorname{argmin}}\left\{E+\sigma_{1} \mathrm{ITT}-\sigma_{3} \mathrm{TTD}\right\}
$$

under

$$
\begin{aligned}
& v_{f} \in \mathcal{U}_{v}=\left\{v_{f}: v_{\min } \leq v_{f} \leq v_{\max }\right\} \\
& l \in \mathcal{U}_{l}=\{l: 0 \leq l \leq L\}
\end{aligned}
$$

\section{B. Simulation results}

In the following the cost functions of Problem 2 are normalized to their respective maximum value, for the sake of comparison. The selection of the weights $\sigma$ represents an additional degree of freedom, depending on the priority one is willing to give to each cost function in the overall objective function. Although the main interest of this work is the energy consumption reduction, the weights choice

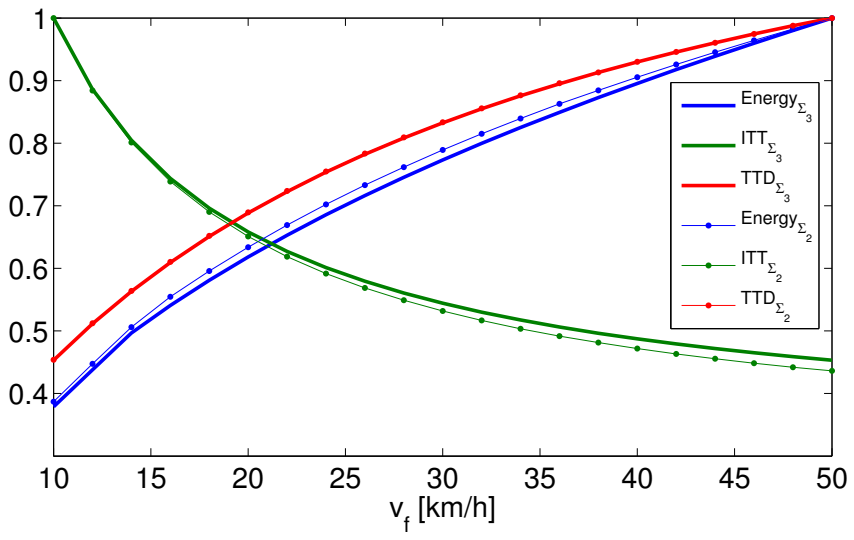

Fig. 4: Energy consumption, ITT and TTD normalized and represented as a function of $v_{f}$. Cost functions for both system $\Sigma_{2}$ and $\Sigma_{3}$ are reported in the graph.

TABLE I: Simulation parameters

\begin{tabular}{c|c|c|c}
\hline Parameter & Description & Value & Unit \\
\hline$L$ & section length & 300 & $\mathrm{~m}$ \\
$w$ & congestion propagation speed & 21.6 & $\mathrm{~km} / \mathrm{h}$ \\
$v_{\max }$ & max velocity & 50 & $\mathrm{~km} / \mathrm{h}$ \\
$v_{\min }$ & min velocity & 10 & $\mathrm{~km} / \mathrm{h}$ \\
$\rho_{m}$ & jam density & 133 & $\mathrm{veh} / \mathrm{km}$ \\
$\bar{\alpha}$ & traffic light split ratio & $1 / 3$ & \\
$a_{\max }$ & max acceleration & 3 & $\mathrm{~m} / \mathrm{s}^{2}$ \\
$\Delta k$ & discretization step & 1 & $\mathrm{~s}$ \\
$T$ & simulation horizon & 600 & $\mathrm{~s}$ \\
$\rho_{f}^{0}$ & initial condition for $\rho_{f}$ & 10 & $\mathrm{veh} / \mathrm{km}$ \\
$\rho_{c}^{0}$ & initial condition for $\rho_{c}$ & 120 & $\mathrm{veh} / \mathrm{km}$ \\
$l^{0}$ & initial condition for $l$ & 200 & $\mathrm{~m}$ \\
\hline
\end{tabular}

$\left(\sigma_{1}, \sigma_{3}\right)=(1.2,0.2)$ was made to give a higher priority to the ITT, in order not to penalize much the traveling time; the TTD is only lightly weighted, so that the energy cost still has importance in the optimization problem.

In Fig.4 it is shown how the difference between the cost functions computed on systems $\Sigma_{2}$ and $\Sigma_{3}$ is minor, proving that the simplification introduced with the averaging method does not compromise the analysis. In simulation it is possible to obtain the total cost of Problem 2 as a function of the only variable speed limit $v_{f}$, and it results to be convex within the constraints interval with a local minimum. It is found that $v_{f}^{*}=26 \mathrm{~km} / \mathrm{h}$ (see Fig.(5)) solves Problem 2, resulting in a reduction of energy consumption of about $29 \%$, paying in terms of ITT which increases by $27 \%$ and of TTD which decreases by $22 \%$, with respect to the case $v_{f}=v_{\max }$. The total objective function of Problem 2 is reduced by $17 \%$ w.r.t. the worst choice of $v_{f}$, and by $6.5 \%$ w.r.t. the standard speed limit of $50 \mathrm{~km} / \mathrm{h}$.

It is natural at this point to apply the optimal velocity $v_{f}^{*}$ that solves Problem 1 for system $\Sigma_{3}$, to the original system $\Sigma_{2}$ in which the traffic lights are modeled with the switching variable $\alpha$. Imposing the same hypothesis of equal boundary flows, achievable by using traffic lights with same phase and cycle times, it is possible to compare the time evolution of 


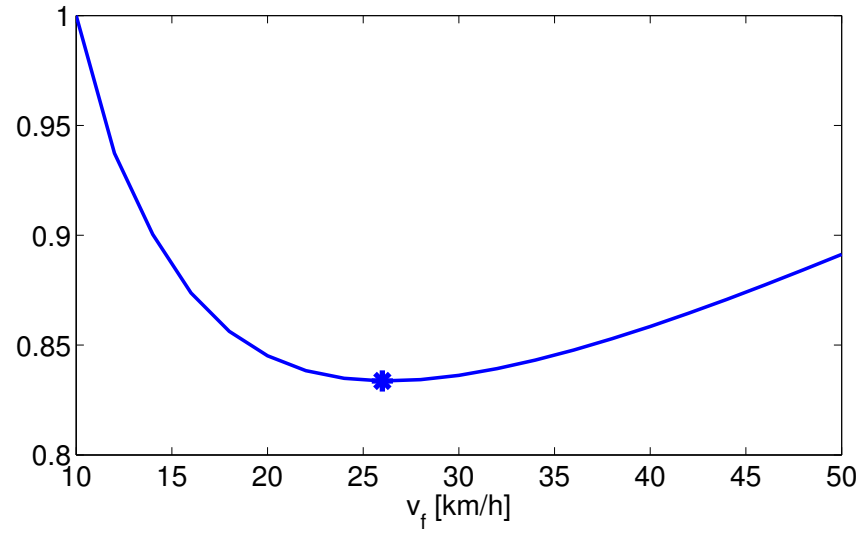

Fig. 5: Overall cost function of Problem 2 for system $\Sigma_{3}$. A local minimum is found at the speed limit $v_{f}^{*}$.
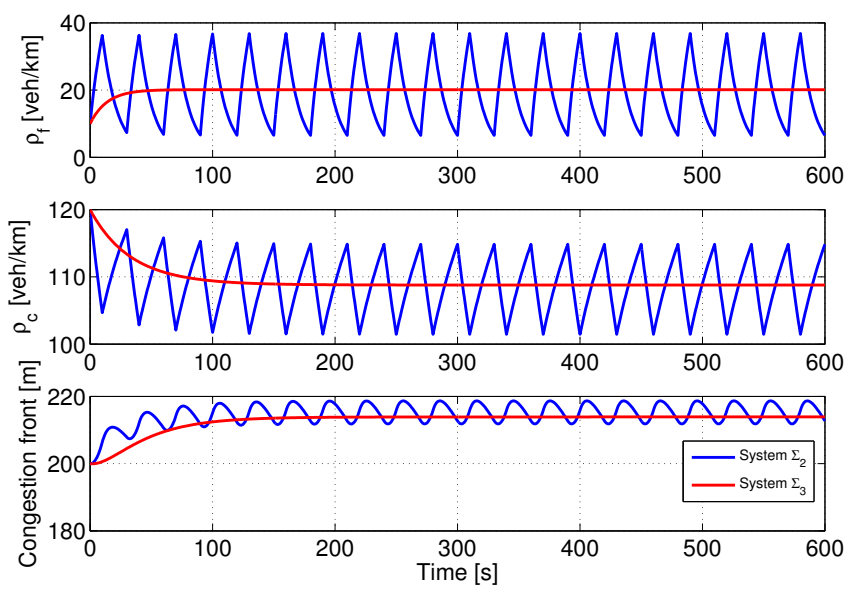

Fig. 6: Time evolution of the two systems $\Sigma_{2}$ and $\Sigma_{3}$ for the optimal value of velocity $v_{f}^{*}$.

the state variables of $\Sigma_{2}$ and $\Sigma_{3}$ and note how the average system $\Sigma_{3}$ tracks closely the true average of $\Sigma_{2}$ (see Fig.6). Using the proposed energy cost function, which is insensitive to temporal accelerations, system $\Sigma_{2}$ appears to be more energy-expensive by only $1.5 \%$ for $v_{f}^{*}=26 \mathrm{~km} / \mathrm{h}$. ITT on $\Sigma_{2}$ is smaller by $1 \%$, while TTD is almost identical.

\section{COnClusions}

In this work a new version of the Variable Length Model, adapted to the urban framework and regulation via variable speed limits, has been employed to simulate traffic flow evolution. Macroscopic traffic performance metrics have been defined to assess the behavior of the system at steadystate and, under the conditions imposed for the solution of Problem 1, it has been shown that there exists a velocity $v_{f}^{*}$ that minimizes the total objective function, resulting in a trade-off between energy consumption reduction and penalization of ITT and TTD.

Future developments will involve the design of a control strategy to track the optimal velocity both in the case of equal boundary flows and in presence of fluctuations in the inflow. The controller should be able to increase the responsiveness of the system to the speed advisory and guarantee robustness to variations in the upstream demand, by limiting the excessive inflow via variable speed limits. The macroscopic energy consumption model could be improved for assessment of $\Sigma_{2}$ by taking into account also temporal accelerations besides spatial ones. Moreover, it is interesting to look at the effects of concatenation of successive sections on the selection of the optimal speed limit. Validation of the model and the eco-driving strategy in a microscopic simulator is also of primary importance.

\section{REFERENCES}

[1] C. Canudas de Wit, "Best-effort highway traffic congestion control via variable speed limits," in IEEE Conference on Decision and Control and European Control Conference, 2011.

[2] C. Canudas de Wit and A. Ferrara, "A New Variable-length Cell Model for Traffic Systems," http://hal.archives-ouvertes.fr/hal-00966968, Internal, Tech. Rep., 2014.

[3] C. F. Daganzo and N. Geroliminis, "An analytical approximation for the macroscopic fundamental diagram of urban traffic," Transportation Research Part B, vol. 42, pp. 771-781, 2008.

[4] S. Lin, B. De Schutter, Y. Xi, and J. Hellendoorn, "A simplified macroscopic urban traffic network model for model-based predictive control," in IFAC Symposium on Transportation Systems, 2009.

[5] M. van den Berg, A. Hegyi, B. De Schutter, and J. Hellendoorn, "A macroscopic traffic flow model for integrated control of freeway and urban traffic networks," in IEEE Conference on Decision and Control, 2003.

[6] C. F. Daganzo, "The cell transmission model: A dynamic representation of highway traffic consistent with the hydrodynamic theory,' Transportation Research Part B, vol. 28, pp. 269-287, 1994.

[7] B. Xie, M. Xu, J. Härri, and Y. Chen, "A Traffic Light Extension to Cell Transmission Model for Estimating Urban Traffic Jam," in IEEE International Symposium on Personal, Indoor and Mobile Radio Communications, 2013.

[8] J. Long, Z. Gao, X. Zhao, A. Lian, and P. Orenstein, "Urban Traffic Jam Simulation Based on the Cell Transmission Model," Networks and Spatial Economics, vol. 11, no. 1, pp. 43-64, 2008.

[9] X. Han, Y. Chen, J. Shi, and Z. He, "An extended cell transmission model based on digraph for urban traffic road network," in IEEE Conference on Intelligent Transportation Systems, 2012.

[10] A. Csikós and I. Varga, "Real-Time Estimation of Emissions Emerging from Motorways Based on Macroscopic Traffic Data," Acta Polytechnica Hungarica, vol. 8, no. 6, pp. 95-110, 2011.

[11] S. K. Zegeye, B. De Schutter, J. Hellendoorn, E. A. Breunesse, and A. Hegyi, "Integrated macroscopic traffic flow, emission, and fuel consumption model for control purposes," Transportation Research Part C, vol. 31, pp. 158-171, 2013.

[12] T. Luspay, B. Kulcsár, I. Varga, S. K. Zegeye, B. De Schutter, and M. Verhaegen, "On acceleration of traffic flow," in IEEE Conference on Intelligent Transportation Systems, 2010.

[13] M. J. Lighthill and G. B. Whitham, "On Kinematic Waves. II. A Theory of Traffic Flow on Long Crowded Roads," Proceedings of the Royal Society A: Mathematical, Physical and Engineering Sciences, vol. 229, no. 1178, pp. 317-345, 1955.

[14] P. I. Richards, "Shock waves on the highway," Operations research, vol. 4, no. 1, pp. 42-51, 1956.

[15] H. K. Khalil, Nonlinear systems, 3rd ed. Prentice Hall, 2002.

[16] K. Aboudolas, M. Papageorgiou, and E. Kosmatopoulos, "Store-andforward based methods for the signal control problem in large-scale congested urban road networks," Transportation Research Part C, vol. 17, pp. 163-174, 2009.

[17] G. De Nunzio, C. Canudas De Wit, P. Moulin, and D. Di Domenico, "Eco-Driving in Urban Traffic Networks using Traffic Signal Information," in IEEE Conference on Decision and Control, 2013.

[18] W. Dib, A. Chasse, A. Sciarretta, and P. Moulin, "Optimal Energy Management Compliant with Online Requirements for an Electric Vehicle in Eco-Driving Applications," in IFAC Workshop on Engine and Powertrain Control, Simulation and Modeling, 2012. 